\title{
Heterogeneity of clinical syndromes related to loss of function mutations in $\mathrm{KCNJ2}$
}

Ionic current abnormalities related to both inherited and acquired arrhythmia syndromes cause sudden cardiac death $[1,2]$. In the heart, ionic current $\mathrm{I}_{\mathrm{K} 1}$ maintains the resting membrane potential and augments terminal repolarization of the action potential [3]. Three inward rectifier channels contribute to cardiac $\mathrm{I}_{\mathrm{K} 1}$, but the dominant component is carried by Kir2.1, encoded by KCNJ2. The importance of this protein is emphasized by the association $\mathrm{KCNJ} 2$ mutations and various inherited arrhythmia syndromes, such as Andersen-Tawil Syndrome (ATS1), Short QT Syndrome 3, and Catecholaminergic Polymorphic Ventricular Tachycardia 3 [46]. The loss of $I_{K 1}$ may lead to arrhythmias by action potential prolongation and subsequent development of early after-depolarization (EAD) and the fatal ventricular arrhythmia Torsade de pointes. Importantly, $\mathrm{I}_{\mathrm{K} 1}$ has also been shown to be down regulated in heart failure (HF) and contributes to the acquired long QT and sudden cardiac death in this disease $[7,8]$. Critical to understanding the pathogenesis of arrhythmia development related to abnormal $\mathrm{I}_{\mathrm{K} 1}$ is the determination of the biophysical mechanisms by which mutations in KCNJ2 affect Kir2.1 function. Here we review the known and hypothesized mechanisms of Kir 2.1 channel dysfunction as it relates to varied clinical syndromes.

ATS1 is an autosomal dominant inherited disease that has a multisystem phenotype spanning the musculoskeletal and cardiac systems [9]. The clinical trial of ATS1 consists of periodic paralysis, ventricular arrhythmias and craniofacial dysmorphic features. Cardiac presentations on ECG reveal prolongation of the QT interval, prominent $\mathrm{U}$ waves with a long QTU, and ventricular ectopy frequently in the form of PVCs, bigeminy, polymorphic VT, and less commonly, bidirectional VT [10]. Dysmorphic features associated with ATS1 include micrognathia, low-set ears, ocular hypertelorism, broad nasal root, clinodactyly and syndactyly $[9,10]$. The diagnosis of ATS is challenging due to incomplete penetrance and varying phenotype severity amongst gene carriers. In one study, gene positive individuals, 58\% expressed the whole clinical triad while at least $81 \%$ had two of the three classical characteristics, leaving $6 \%$ of individuals showing no penetrance of the gene [11]. The specific location of a disease causing variant or hormonal regulation may affect expression variability $[12,13]$.

Predominantly mutations in KCNJ2 are missense or deletion mutations with a variety of downstream effect on Kir2.1 protein function. Phosphatidylinositol 4,5-bisphosphate (PIP2) binding is necessary for Kir2.1 to open [14], and ATS1 has been shown to be a result of KCNJ2 mutations that affect PIP2 binding or an allosteric conformational change leading to decreased Kir2.1 current [15]. Other ATS causing KCNJ2 mutations include disturbances in the pore selectivity filter and misfolded or sequestered proteins, but these make up the minority of disease causing mutations $[15,16]$. The C-terminus of Kir2.1, in addition to PIP2 binding sites, is home to the Kir2.1 endoplasmic reticulum export sequence and is implicated as mechanism for Kir2.1 loss due to trafficking defect [12]. Additionally, channel interacting proteins, such as caveolin-3, modifier genes, and epigenetic factors may play a role in ATS phenotype, penetrance and arrhythmia susceptibility [17-19], but are not well understood.
Matt Kalscheur, Hannah Van Ert \& Lee L Eckhardt*

Department of Cardiology, Soma State Hospital, Soma, Manisa, Turkey School of Medicine and Public Health Madison, University of Wisconsin Madison, WI, United States

*Author for correspondence:

Tel.: +16082631530

Ile@medicine.wisc.edu 
CPVT is a rare arrhythmogenic disorder characterized by adrenergic dependent bidirectional and polymorphic ventricular tachycardia (PMVT). Defining features of the phenotype include a normal resting ECG, including $\mathrm{QT}_{\mathrm{c}}$, and an arrhythmia reproducible with exercise, stress or isoproterenol infusion. Approximately $50-60 \%$ of patients harbor mutations in either the cardiac ryanodine receptor (RYR2, CPVT1) or cardiac calsequestrin 2 (CASQ2, CPVT2) [6,20,21]. The cardiac ryanodine receptor and calsequestrin are intrinsic to cellular calcium homeostasis, and arrhythmia mechanisms may be related to calcium overload/dysregulation and production of delayed after-depolarization [22] or increased automaticity [23]. Interestingly, the ryanodine receptor is also active in pancreatic islet cells and plays a significant role in insulin secretion and cell metabolism [24]. More recently other genes have been associated with a CPVTlike phenotype such as KCNJ2, designated CPVT3, ankyrinB and triadin [6,25-27].

To date, two KCNJ2 mutations associated with CPVT3 have been characterized, V227F and R67Q, by our group $[28,29]$. The patients identified with CPVT3 were female and presented with exertion/ emotion related near-syncope and syncope. The patients demonstrated neither dysmorphic features nor periodic paralysis and had normal $\mathrm{QT}_{c}$ intervals. Both patients did have prominent $U$ waves on resting ECG. Subsequent testing demonstrated exercise-induced arrhythmias that included salvos of polymorphic and bidirectional ventricular tachycardia. Both V227FKir2.1 and R67Q-Kir2.1 demonstrated adrenergic dependent loss of function in heterologous cells $[28,29]$.

There is debate as to whether the KCNJ2-related arrhythmia syndromes classified here as ATS1 and CPVT3 are: (1) two distinct entities with different outcomes and with different underlying molecular and cellular mechanisms, are (2) the same clinical syndrome with variability in presentation as CPVT phenocopies, or (3) if the arrhythmia syndromes associated with KCNJ2 mutations have been unintentionally misclassified for the lack of a better definition for these syndromes. It is

\section{References}

1. Schwartz PJ, Crotti L, Insolia R. Long-QT syndrome: from genetics to management. Circ. Arrhythm. Electrophysiol. 5, 868877 (2012).

2. Boyden PA, Jeck CD. Ion channel function in disease. Cardiovasc. Res. 29, 312-318 (1995).

3. Wang Z, Yue L, White M, Pelletier G, Nattel S. Differential distribution of inward rectifier potassium channel transcripts in human atrium versus ventricle. Circulation. 98, 2422-2428 (1998). clear that there has been considerable overlap between mutations that have been associated with ATS1 and CPVT3 [11,29]. However, supporting two clinical entities includes both the distinct clinical presentation and cellular physiology. As described above, exercise is not a constant trigger for the arrhythmias associated with the clinical phenotype of ATS1, and in some instances, exercise suppresses these arrhythmias $[11,13]$. In contrast, the arrhythmias in patients characterized as having CPVT3 mutations were precipitated by exercise or stress. Additionally, in terms of functional characterization, most Kir2.1 mutations associated with ATS1 exhibit dominant-negative Kir2.1 current when co-expressed with WT-Kir2.1 while the Kir2.1 mutations associated with CPVT3 displayed markedly decreased outward Kir2.1 current only after adrenergic stimulation.

The mechanisms underlying the functional differences observed with these mutations are not clear at this time; however, they are an area of active research for our laboratory and others. Phosphatidylinositol 4,5-bisphosphate (PIP2) is a well-established regulator of inward rectifying potassium channels [14]. Many of the Kir2.1 mutations associated with ATS1 and the R67Q-KCNJ2 mutation associated CPVT3 occur in regions that affect the interaction of the channel with PIP2 [30]. An N-terminus mutation that alters Kir2.1 sensitivity to PIP2 has been shown to exaggerate the inhibition of Kir2.1 by a divalent cation, magnesium [31]. Therefore, one hypothesis for adrenergic modulated channel function may be enhanced inhibition in the presence of increased cellular calcium [29].

Important questions remain in the phenotypegenotype correlation for clinical syndromes related to KCNJ2 mutations. Careful clinical phenotyping as well as functional studies, which focus on the underlying arrhythmia mechanisms, will allow greater clarity for these syndromes. Such an understanding is crucial in providing clinical care and applying concepts of precision medicine for the care of these patients.

4. Plaster NM, Tawil R, Tristani-Firouzi M, et al. Mutations in Kir2.1 cause the developmental and episodic electrical phenotypes of Andersen's syndrome. Cell. 105, 511-519 (2001).

5. Priori SG, Pandit SV, Rivolta I, et al. A novel form of short QT syndrome (SQT3) is caused by a mutation in the KCNJ2 gene. Circ. Res. 96, 800-807 (2005).

6. Tester D, Arya P, Will M, et al. Genotypic heterogeneity and phenotypic mimicry among unrelated patients referred for catecholaminergic polymorphic ventricular tachycardia genetic testing. Heart. Rhythm. 3, 800-805 (2006). 
7. Beuckelmann DJ, Nabauer M, Erdmann E. Alterations of K+ currents in isolated human ventricular myocytes from patients with terminal heart failure. Circulation. Research. 73, 379-385 (1993).

8. Tomaselli GF, Beuckelmann DJ, Calkins HG, et al. Sudden cardiac death in heart failure. The role of abnormal repolarization. Circulation. 90, 2534-2539 (1994).

9. Andersen ED, Krasilnikoff PA, Overvad H. Intermittent muscular weakness, extrasystoles, and multiple developmental anomalies. A new syndrome? Acta. Paediatrica. 60, 559-564 (1971).

10. Tristani-Firouzi M, Jensen JL, Donaldson MR, et al. (2002) Functional and clinical characterization of KCNJ2 mutations associated with LQT7 (Andersen syndrome). J. Clin. Invest. 110, 381-388.

11. Tristani-Firouzi M, Etheridge SP. Kir 2.1 channelopathies: the Andersen-Tawil syndrome. Pflugers. Arch. 460: 289-294 (2010).

12. Kimura $\mathrm{H}$, Zhou J, Kawamura M, et al. Phenotype variability in patients carrying KCNJ2 mutations. Circ. Cardiovasc. Genet. 5, 344-353 (2012).

13. Andelfinger G, Tapper AR, Welch RC, et al. KCNJ2 mutation results in Andersen syndrome with sex-specific cardiac and skeletal muscle phenotypes. Am. J. Hum. Genet. 71, 663-668 (2002).

14. Fan Z, Makielski JC. Anionic phospholipids activate ATPsensitive potassium channels. J. Biol. Chem. 272, 5388-5395 (1997).

15. Donaldson MR, Jensen JL, Tristani-Firouzi M, et al. PIP2 binding residues of Kir2.1 are common targets of mutations causing Andersen syndrome. Neurology. 60, 1811-1816 (2003).

16. Nguyen HL, Pieper GH, Wilders R. Andersen-Tawil syndrome: clinical and molecular aspects. Int. J. Cardiol. 170, 1-16 (2013).

17. Vaidyanathan R, O'Connell RP, Deo M, et al. The ionic bases of the action potential in isolated mouse cardiac Purkinje cell. Heart. Rhythm. 10, 80-87 (2013).

18. Imboden M, Swan H, Denjoy I, et al. (2006) Female predominance and transmission distortion in the long- QT syndrome. N. Engl. J. Med. 355, 2744-2751 (2006).

19. Sardu C, Santamaria M, Paolisso G, Marfella R. microRNA expression changes after atrial fibrillation catheter ablation. Pharmacogenomics. 16, 1863-1877 (2015).
20. Leenhardt A, Lucet V, Denjoy I, et al. Catecholaminergic polymorphic ventricular tachycardia in children. A 7- year follow-up of 21 patients. Circulation. 91, 1512-1519 (1995).

21. Priori SG, Napolitano C, Tiso N, et al. Mutations in the cardiac ryanodine receptor gene (hRyR2) underlie catecholaminergic polymorphic ventricular tachycardia. Circulation. 103, 196-200 (2001).

22. Cerrone M, Noujaim SF, Tolkacheva EG, et al. Arrhythmogenic mechanisms in a mouse model of catecholaminergic polymorphic ventricular tachycardia. Circulation. Research. 101: 1039-1048 (2007).

23. Liu N, Denegri M, Ruan Y, Avelino-Cruz JE, Perissi A, et al. Short communication: flecainide exerts an antiarrhythmic effect in a mouse model of catecholaminergic polymorphic ventricular tachycardia by increasing the threshold for triggered activity. Circulation. Research. 109, 291-295 (2011).

24. Santulli G, Pagano G, Sardu C, et al. Calcium release channel RyR2 regulates insulin release and glucose homeostasis. J. Clin. Invest. 125, 1968-1978 (2015).

25. Mohler PJ, Schott JJ, Gramolini AO, et al. Ankyrin-B mutation causes type 4 long-QT cardiac arrhythmia and sudden cardiac death. Nature. 421, 634-639 (2003).

26. Chopra N, Knollmann BC. Triadin regulates cardiac muscle couplon structure and microdomain $\mathrm{Ca}^{(2+}$ signalling: a path towards ventricular arrhythmias. Cardiovasc. Res. 98, 187-191 (2013).

27. Kirchhof P, Klimas J, Fabritz L, et al. Stress and high heart rate provoke ventricular tachycardia in mice expressing triadin. $J$. Mol. Cell. Cardiol. 42, 962-971 (2007).

28. Vega AL, Tester DJ, Ackerman MJ, Makielski JC. Protein kinase A- dependent biophysical phenotype for V227F-KCNJ2 mutation in catecholaminergic polymorphic ventricular tachycardia. Circ. Arrhythm. Electrophysiol. 2, 540-547 (2009).

29. Kalscheur MM, Vaidyanathan R, Orland KM, et al. KCNJ2 mutation causes an adrenergic-dependent rectification abnormality with calcium sensitivity and ventricular arrhythmia. Heart. Rhythm. 11, 885-894 (2014).

30. Lopes $\mathrm{CM}$, Zhang $\mathrm{H}$, Rohacs $\mathrm{T}$, et al. Alterations in conserved Kir channel-PIP2 interactions underlie channelopathies. Neuron. 34, 933-944 (2002).

31. Ballester LY, Vanoye CG, George AL. Exaggerated Mg2+ inhibition of Kir2.1 as a consequence of reduced PIP2 sensitivity in Andersen syndrome. Channels (Austin). 1, 209217 (2007). 